\title{
FUSED SILICA MICRO BIRDBATH SHELL RESONATORS WITH 1.2 MILLION Q AND 43 SECOND DECAY TIME CONSTANT
}

\author{
J. Cho, T. Nagourney, A. Darvishian, B. Shiari, J.-K. Woo, and K. Najafi
}

Center for Wireless Integrated MicroSensing and Systems, University of Michigan, USA

\begin{abstract}
We report a micro-scale fused silica (FS) birdbath (BB) shell resonator with a high mechanical quality factor $(Q=1.2$ million), long decay time constant ( $\tau=43.6 \mathrm{~s}$ ), a resonant frequency of 8.8 $\mathrm{kHz}$ for wineglass modes, and excellent symmetry resulting in a frequency split of only $6.7 \mathrm{~Hz}$ between two wineglass modes. The resonator has a volume of $41 \mathrm{~mm}^{3}$ (radius $=2.5 \mathrm{~mm}$, anchor radius $=0.5 \mathrm{~mm}$, height $\sim 2.1 \mathrm{~mm}$ ). The resonator is useful in a wide range of applications, including inertial sensors, timing devices, and chemical and biological sensors.
\end{abstract}

\section{INTRODUCTION}

A high $Q$ and long decay time constant $(\tau)$ are desired in many sensing applications, because they reduce mechanical noise and bias drift. A silicon gyroscope with $f \sim 2 \mathrm{kHz}$ and $Q$ of 1.16 million has been reported [1]. Macro-scale FS wineglass resonators with $Q$ of tens of millions at $3 \mathrm{kHz}$ [2] or 1 million at $>70 \mathrm{kHz}$ have been reported [3]. For micro vibratory gyroscopes, an $f$ of $\sim 10 \mathrm{kHz}$ is preferred, because the sensor 1) can operate above the $f$ of environmental noises, 2) has good flexibility, which results in a large scale factor, and 3 ) and has a long decay time constant $(\tau)$ leading to good bias stability. On the micro scale, there has been a lack of materials and fabrication techniques to achieve an ultrahigh $Q$ and a long $\tau$ in this frequency range. It is difficult to achieve $>1$ million $Q$ with $\mathrm{Si}$ in the $\sim 10 \mathrm{kHz}$ frequency range due to high thermoelastic damping (TED). FS suffers less TED than Si; however, it is difficult to etch or form complicated FS structures using conventional fabrication processes. We overcame these challenges and achieved both very high $Q$, long $\tau$, and very small frequency split $(\Delta f)$ in our birdbath shell resonators.

\section{FABRICATION}

We fabricated our shells using a 3D micro blowtorching process (Figure 1) [4]. A thin FS substrate (thickness $\sim 100 \mu \mathrm{m}$ ) is placed on top of a graphite mold and is controllably heated by a propane-oxygen blowtorch at $>1600{ }^{\circ} \mathrm{C}$ while applying vacuum from the bottom of the mold. The shells were released using conventional lapping and CMP processes. The devices have a 2.5 $\mathrm{mm}$ radius, $0.5 \mathrm{~mm}$ anchor radius, $\sim 2.1 \mathrm{~mm}$ height (Fig. 2), and $\sim 60 \mu \mathrm{m}$ rim thickness (Figure 2).

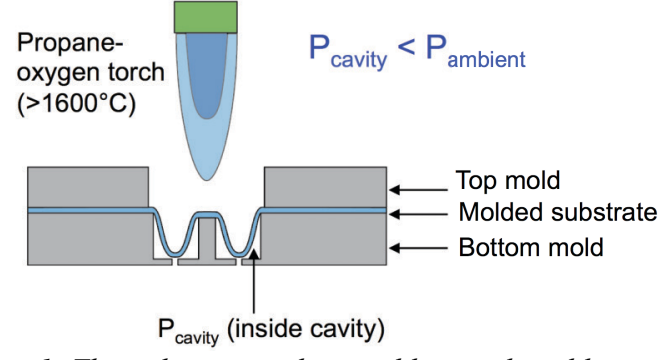

Figure 1: Three dimensional micro blowtorch molding process

\section{DEVICE CHARACTERIZATION}

The $f$ and $\tau$ of an uncoated BB resonator were measured by
AS\&T using conformal Laser Doppler Vibrometry [5] while driving it with a PZT actuator. We tested a metal-coated BB resonator (sputtered $\mathrm{Cr} / \mathrm{Au}=15 / 50 \AA$ ) using a capacitive measurement method. Both tests were done at $<1 \mathrm{mTorr}$ pressure at room temperature. Both samples were cleaned using BHF for 3$30 \mathrm{~s}$ before testing. The uncoated $\mathrm{BB}$ resonator has $n=2$ wineglass modes at $8.718 \mathrm{kHz}$ with $\tau=42.18 \mathrm{~s}(Q=1.16$ million $)$ and 8.725 $\mathrm{kHz}$ with $\tau=43.58 \mathrm{~s}(Q=1.19$ million) (Figure 3$)$. These time constants are the longest reported for small wineglass structures. The coated BB resonator has wineglass modes at $10.163 \mathrm{kHz}$ with $\tau=15.24 \mathrm{~s}(Q=486 \mathrm{k})$ and $10.188 \mathrm{kHz}$ with $\tau=9.70 \mathrm{~s}(Q=310 \mathrm{k})$ (Figure 4). We believe that a combination of large device height, BHF etching, and a thinner metal layer led to the improvement of $\tau$. Figure 5 shows the dependency of $\tau$ on the BHF etching duration as well as the thicknesses of the metal coating on six different devices. We found $\sim 2 \times$ improvement in $\tau$ due to the reduced metal thickness and $30 \mathrm{~s}$ BHF etching. However, after longer BHF etching ( $95 \mathrm{~s}$ ), we found a reduction in $\tau$ even with the same metal thickness, likely due to increase in surface roughness.

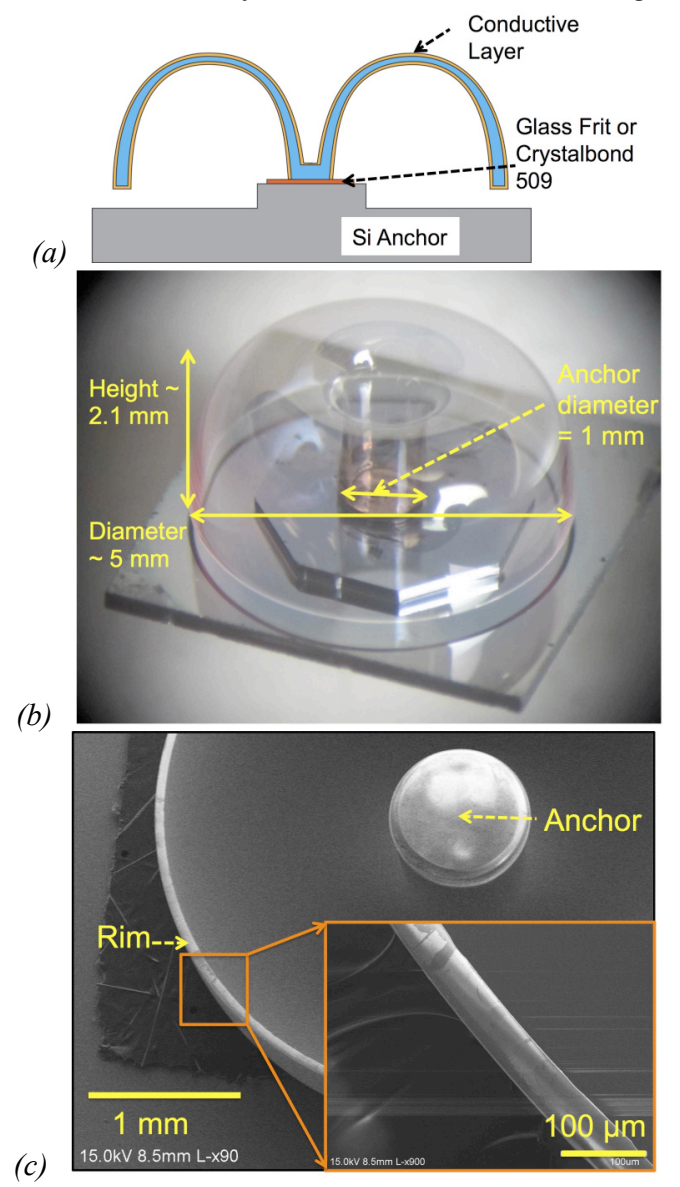

Figure 2: (a) Illustration, (b) photograph, and (c) SEM image of fused silica micro birdbath shell resonator. The shell is coated with $\mathrm{Cr} / \mathrm{Au}=15 / 50 \mathrm{~A}$. Device dimensions: radius $\sim 2.5 \mathrm{~mm}$, anchor radius $=0.5 \mathrm{~mm}$, height $\sim 2.1 \mathrm{~mm}$, rim thickness $\sim 60 \mu \mathrm{m}$. 


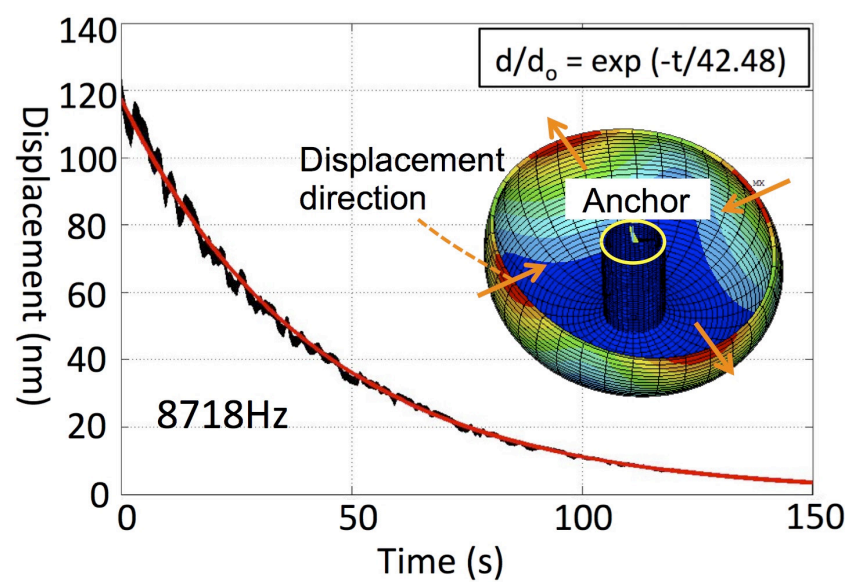

(a)

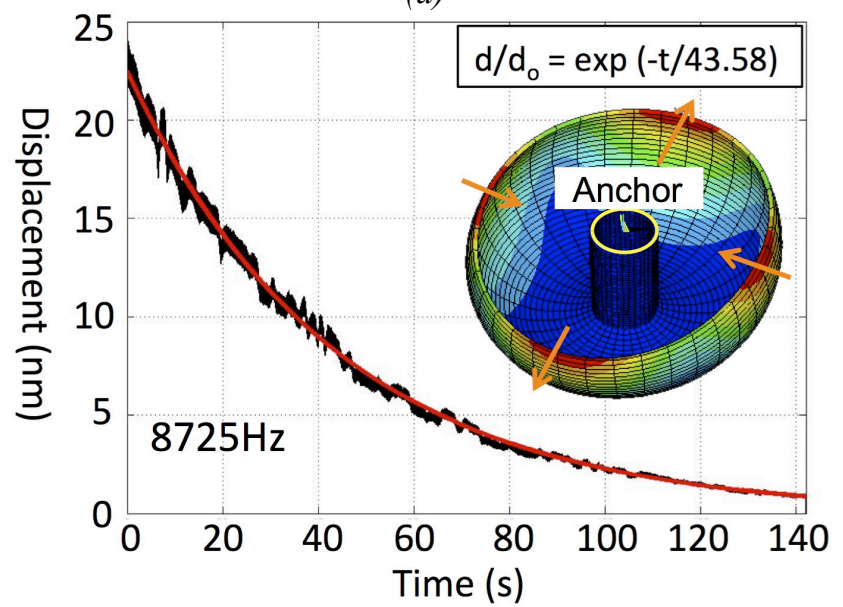

(b)

Figure 3: Ring-down time plot of $n=2$ wineglass modes of uncoated $B B$ resonator using conformal Laser Doppler Vibrometry: (a) $f=8.718 \mathrm{kHz}$ and $\tau=42.18 \mathrm{~s}(Q=1.16$ million). (b) $f=8.725 \mathrm{kHz}$ and $\tau=43.58 \mathrm{~s}(Q=1.19$ million) [5].

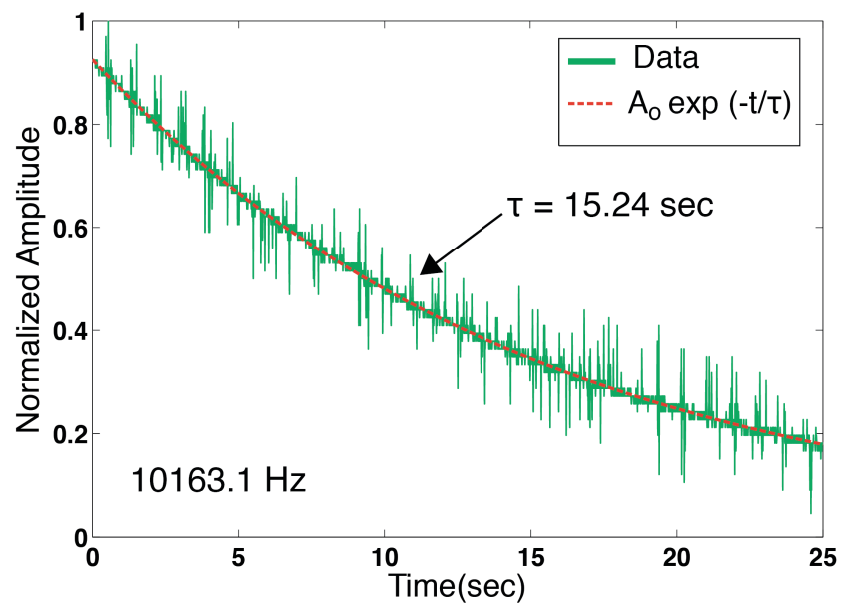

Figure 4: Ring-down time plot of $n=2$ wineglass modes of coated $B B$ resonator using capacitive measurement method: $f=10.163 \mathrm{kHz}$ and $\tau=15.24 \mathrm{~s}(Q=486 \mathrm{k})$. Another mode has $f=10.188 \mathrm{kHz}$ and $\tau=9.70 \mathrm{~s}(Q=$ $310 k)$.

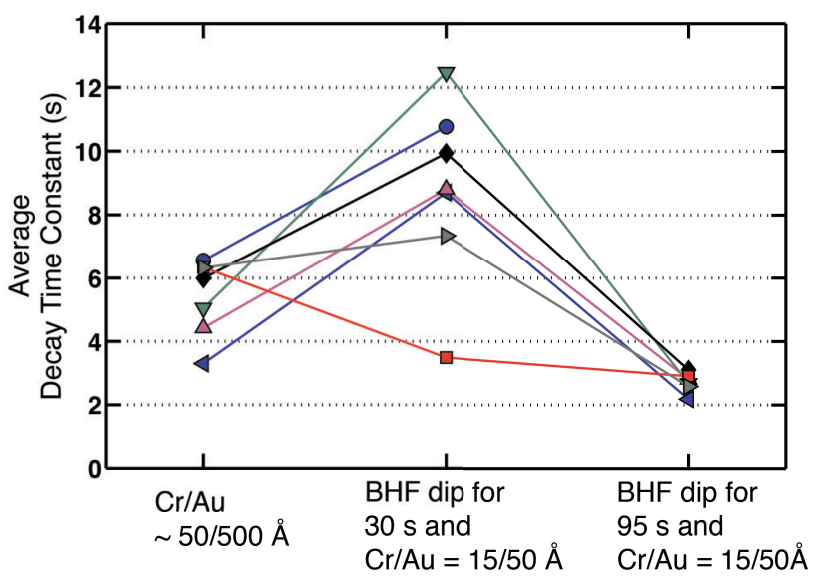

Process Conditions

Figure 5: Dependency of $\tau$ on metal coating and BHF etching for six different samples. These devices have resonant frequencies at $9.5 \sim 11 \mathrm{kHz}$.

\section{CONCLUSION}

We report a micro BB resonator with a small volume $(\sim 41$ $\left.\mathrm{mm}^{3}\right)$ with one of the best $Q \mathrm{~s}$ (=1.2 million), $\tau \mathrm{s}(=43.58 \mathrm{~s})$, and structural symmetries $(\Delta f=7 \mathrm{~Hz})$. The good resonance characteristics are due to the low TED and ultra-low surface roughness $(<1 \mathrm{~nm})$ achieved using the 3-D micro blowtorch molding process. The process can be adopted to fabricate a wider variety of high-performance micro sensors and actuators. We found strong dependency of $\tau$ on the conductive metal coating and surface cleaning.

\section{ACKNOWLEGEMENTS}

This work is supported by DARPA MRIG award \#W31P4Q11-1-0002. We thank Jim Kilpatrick, Adela Apostol, and Vladimir Markov of Advanced Systems and Technologies, Inc., Irvine, CA for measurements with the conformal imaging vibrometry. We thank Dr. Radheshyam Tewari and Professor Craig Friedrich at Michigan Technological University for machining graphite molds. Portions of this work were done in the Lurie Nanofabrication Facility (LNF), a site of the National Nanotechnology Infrastructure Network (NNIN), which is supported in part by the National Science Foundation (NSF).

\section{REFERENCES}

[1] S.A. Zotov, A. A. Trusov, A. M. Shkel, "High-Range Angular Rate Sensor Based on Mechanical Frequency Modulation”, J. Microelectromech. Sys., 22, 398 (2012).

[2] E. J. Loper and D. D. Lynch, "Projected System Performance Based on Recent HRG Test Results", Proc. IEEE/AIAA 5th Digital Avionics System Conference, Seattle, WA (1983), pp. 18.1.1 - 18.1.6.

[3] M. J. Ahamed, D. Senkal, and A. M. Shkel, "Effect of Annealing on Mechanical Quality Factor of Fused Quartz Hemispherical Resonator", Proc. IEEE ISSIS 2014, Laguna Beach, CA (2014), pp. 59-62.

[4] J. Cho, J. Yan, J. A. Gregory, H. Eberhart, R. L. Peterson, and K. Najafi, "3-Dimensional Blow Torch-Molding of Fused Silica Microstructures”, J. Microelectromech. Sys., 22, 1276 (2013).

[5] J. Kilpatrick, A. Apostol, and V. Markov, "MEMS characterization with Conformal Imaging Vibrometry", Proc. IEEE ISISS 2014, Laguna Beach, CA (2014), pp. 86-88. 